Antonio Carlos VIerRa Lopes'

KLeBer PIMENTEL ${ }^{2}$

Alessandro de Moura Almeida ${ }^{3}$

Eduardo Costa Matos ${ }^{3}$

Maria Betânia Pereira Toralles ${ }^{4}$

Artigos originais

Palavras-chaves

Amostra da vilosidade coriônica/ efeitos adversos

Diagnóstico pré-nata Anormalidades/diagnóstico Aberrações cromossômicas Cromossomos humanos par 21 Síndrome de Down

Keywords

Chorionic villi sampling/ adverse effects

Prenatal diagnosis

Abnormalities/diagnosis

Chromosome aberrations Chromosomes, human, pair 21 Down Syndrome

\section{Complicações materno-fetais da biópsia de vilo corial: experiência de um centro especializado do Nordeste do Brasil}

\author{
Fetal and maternal complications of chorionic villus sampling: \\ results from a specialized center in the Northeast of Brazil
}

\title{
Resumo
}

OBJETIVO: avaliar complicações maternas e fetais após realização de biópsia de vilo corial (BVC) para diagnóstico pré-natal de alterações genéticas, na cidade de Salvador (BA). MÉTODOS: série de 958 gestantes de risco para cromossomopatias, submetidas à BVC realizada entre a nona e a $24^{a}$ semanas de gestação, por via transabdominal, utilizando agulha espinhal 18G 31/2, guiada por ultra-sonografia, entre 1990 e 2006. As variáveis para a análise de complicações imediatas foram cólicas uterinas, hematoma subcoriônico, punção acidental da cavidade amniótica, dor no local da punção, amniorrexe, desconforto abdominal, bradicardia fetal e sangramento vaginal, e para complicações tardias, dor abdominal, sangramento vaginal, amniorrexe, infecção e abortamento espontâneo. Complicações obstétricas e fetais (parto prematuro, descolamento prematuro de placenta, placenta prévia e malformações anatômicas fetais) foram também estudadas. Para análise estatística, utilizaram-se $\circ \chi^{2}$ e $\circ$ teste $t$ de Student ou Mann-Whitney; o nível

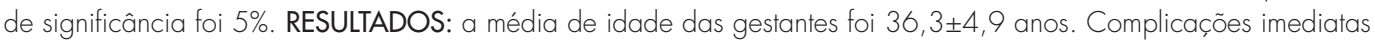
foram encontradas em 182 (19\%) casos (cólica uterina em 14\%, hematoma subcoriônico em 1,8\% e punção amniótica acidental em 1,3\%) e tardias em 32 (3,3\%) casos (sangramento vaginal em 1,6\%, dor abdominal em 1,4\%, amniorrexe em $0,3 \%$ e aborto espontâneo em 1,6\%). Não foi observado descolamento prematuro de placenta, placenta prévia ou malformação fetal. CONCLUSÕES: a BVC revelou-se procedimento simples e seguro. A BVC pode ser utilizada em gestantes que necessitam de diagnóstico pré-natal devido ao risco de anomalias genéticas.

\section{Abstract}

PURPOSE: to evaluate fetal maternal complications after chorionic villus sampling (CVS) for prenatal diagnosis of genetic disorders in pregnant women of Salvador (BA), Brazil. METHODS: case-series study of 958 pregnancies with high risk for chromosomal abnormality submitted to CVS transabdominal between the ninth to the $24^{\text {th }}$ week of gestation, using an ultrasound-guided $18 \mathrm{G} 3^{1 / 2}$ spinal needle, from 1990 to 2006 . The variables for the analysis of immediate complications were uterine cramps, subchorionic hematoma, accidental amniotic cavity punction, pain in the punction area, amniotic fluid leakage, abdominal discomfort, fetal arrhythmias and vaginal bleeding, and of late complication, abdominal pain, vaginal bleeding, amniotic fluid leakage, infection and spontaneous miscarriage. Premature labor, obstetrical complications (abruption placenta and placenta previa) and newborn malformation were also studied. Quisquare, Student's " $t$ " or Mann-Whitney tests were used for the statistical analysis; the significance level was 5\%. RESULTS: maternal mean age was $36.3 \pm 4.9$ years old. Immediate complications ware found in $182(19 \%$ ) cases (uterine cramp in $14 \%$, subchorionic hematoma in $1.8 \%$ and accidental amniotic cavity punction in $1.3 \%$. Late complications were found in $32(3.3 \%$ ) cases (vaginal bleeding in $1.6 \%$, abdominal pain in $1.4 \%$, amniotic fluid leakage in $0.3 \%$ and spontaneous miscarriage in $1.6 \%$ cases). There was no case of abruption placentae, placenta previa or fetal malformation. CONCLUSIONS: CVS is a simple and safe procedure. CVS should be performed in high risk pregnant patients who need prenatal diagnosis of fetal chromosomal abnormalities.

Correspondência:

Antonio Carlos Vieira Lopes Rua Dr. José Carlos, 137, apto. 501 Condomínio Edifício Parque das Mangueiras - Acupe de Brotas CEP 40290-040 - Salvador/BA

Recebido $14 / 05 / 2007$

Aceito com modificacọoes $04 / 07 / 2007$
Maternidade Climério de Oliveira da Faculdade de Medicina da Universidade Federal da Bahia - MCO-UFBA - Salvador (BA), Brasil; Clínica Dr. Antonio Carlos Vieira Lopes - Salvador (BA), Brasil.

' Professor Adjunto; Chefe do Departamento de Ginecologia, Obstetrícia e Reprodução Humana da Faculdade de Medicina da Universidade Federal da Bahia - UFBA - Salvador (BA), Brasil.

2 Professor Assistente da Escola Baiana de Medicina e Saúde Pública - EBMSP - Salvador (BA), Brasil; Universidade Católica do Salvador - UCSAL - Salvador (BA), Brasil.

${ }^{3}$ Acadêmico do Programa de Educação Tutorial da Faculdade de Medicina da Universidade Federal da Bahia - UFBA - Salvador (BA), Brasil.

4 Professora Adjunta de Genética do Departamento de Pediatria da Faculdade de Medicina da Universidade Federal da Bahia - UFBA - Salvador (BA), Brasil. 


\section{Introdução}

O diagnóstico pré-natal com o estudo do cariótipo fetal e testes genéticos continua sendo realizado por meio de métodos invasivos, predominantemente amniocentese clássica (AMC) entre a $16^{\mathrm{a}}$ e a $20^{\mathrm{a}}$ semanas e biópsia de vilo corial (BVC) entre a $10^{\mathrm{a}}$ e a $12^{\mathrm{a}}$ semanas $^{1}$. A recente descoberta da presença de DNA fetal no plasma materno representa possibilidade futura do desenvolvimento de técnica não invasiva para diagnóstico de doenças fetais ${ }^{2}$.

A principal indicação para ambos os procedimentos (AMC e BVC) tem sido a idade materna avançada (igual ou superior a 35 anos), por estar associada a risco elevado de anormalidades cromossômicas fetais ${ }^{3}$. Outras indicações freqüentes são alterações dos testes de rastreamento pré-natal de trissomia, ocorrência de anormalidade cromossômica em gravidez anterior, identificação de marcadores fetais de cromossomopatias pela ultra-sonografia, ansiedade do casal e teste de paternidade ${ }^{4,5}$.

A BVC foi introduzida na prática médica em 1980, sendo inicialmente realizada a partir da sexta semana de gravidez e por via transcervical (BVC-TC) ${ }^{6,7}$. Posteriormente, a via transabdominal (BVC-TA) foi adotada como alternativa, objetivando reduzir os riscos de complicações, sobretudo de infecção e interrupção da gravidez 6 .

Foi descrito que a amniocentese precoce (AMP) acarreta maior risco de abortamento espontâneo (4,4 versus $2,3 \%)$ e pé torto congênito (1,8 versus $0,2 \%)$ quando comparada com a BVC-TA, realizadas no mesmo período $^{8}$. Estudo randomizado comparando BVC-TC, BVC-TA e AMC revela taxas de perdas gestacionais totais de 10,9, 6,3 e 6,4\%, respectivamente. Quando comparados os dois procedimentos (BVC-TC e BVC-TA), as taxas de perdas gestacionais espontâneas foram de 7,7 e 3,7\%, respectivamente ${ }^{9}$. Em publicação mais recente, comparando a AMC e a BVC, todavia sem indicação da via de abordagem, as taxas de perdas gestacionais nos dois grupos foram semelhantes nos últimos cinco anos do estudo (1998 a 2003) $)^{10}$. Os resultados de um estudo randomizado internacional incluindo 3.775 casos entre 11 e 14 semanas demonstraram ser a BVC-TA um método mais seguro que a AMP, estando esta última associada a maior risco de pé torto congênito e interrupção da gravidez ${ }^{11}$.

No início de 1990, foi descrito uma possível associação de BVC com defeitos congênitos em membro de recém-nascido submetido a este procedimento na nona semana de gravidez ${ }^{12}$. Mais tarde, diversos relatos foram publicados identificando a BVC como causa das malformações. Em 1992, a Organização Mundial da Saúde (OMS) analisou os dados de 138.996 gravidezes submetidas à BVC, revelando um total de 77 fetos e recém-nascidos com defeitos de membros $(64,6 \%$ de membros superiores, $12,5 \%$ inferiores e $20,8 \%$ em ambos $)^{13}$. Os resultados encontrados, no entanto, estavam de acordo com a distribuição de defeitos de membros fetais em diversos estudos populacionais. Os autores concluíram que não havia evidências de aumento do risco de defeitos de membros fetais após a realização de BVC, bem como não existia associação entre a idade gestacional e a gravidade da doença ${ }^{14}$.

Com relação a complicações maternas, existem poucos relatos, sendo relacionados perda de líquido amniótico, hemorragias obstétricas tardias e corioamnionite ${ }^{9}$ que, entretanto, não foram confirmadas por outros autores ${ }^{15}$.

Embora a BVC seja considerada, na atualidade, um procedimento seguro pela baixa freqüência de complicações ${ }^{16}$, é um método dependente da habilidade do operador e da curva de aprendizado ${ }^{6,10,15,17}$. O verdadeiro risco da BVC ainda não é bem definido, sobretudo pela inexistência de trabalhos randomizados, comparando pacientes expostas ao procedimento com não expostas. A maioria das publicações tem comparado a BVC com a AMC do segundo trimestre, ou com a AMP, realizada na mesma época da $\mathrm{BVC}^{11,18}$. As publicações nacionais sobre o assunto são escassas, alguns com casuísticas pequenas e publicadas há quase duas décadas ${ }^{19,20}$. O objetivo desse trabalho foi avaliar as complicações maternas e fetais decorrentes da realização de BVC-TA, com a finalidade de conhecer a segurança de sua aplicabilidade na prática obstétrica como método de diagnóstico fetal.

\section{Métodos}

O desenho de estudo é uma série de casos com 958 gestantes com gravidezes únicas, submetidas à BVC-TA para diagnóstico pré-natal, no período de 1990 a 2006, em serviço de referência para Medicina Fetal, Clínica Dr. Antonio Carlos Vieira Lopes, e na Maternidade Climério de Oliveira da Universidade Federal da Bahia (MCO-UFBA). Os dados foram coletados em formulário criado e padronizado para o estudo. Todas as pacientes concordaram em assinar um termo de consentimento livre e esclarecido e o protocolo de pesquisa foi submetido e aprovado pelo Comitê de Ética em Pesquisa da MCO-UFBA.

A média de idade das pacientes foi $36,3 \pm$ 4,9 anos, com limites de 15 e 47 anos, e a mediana, 37 anos. As medianas do número de gestações, partos 
e abortos foram 2, 1 e 0 , e a moda 2,0 e 0 , respectivamente. Duzentos e oitenta e nove pacientes $(30,2 \%)$ eram primigestas, $320(33,4 \%)$ secundigestas e 349 $(36,4 \%)$ tinham engravidado três ou mais vezes. A média da idade gestacional quando da realização da BVC foi $13,0 \pm 2,2$ semanas, sendo a mediana e a moda de 12 semanas. As principais indicações para o procedimento incluíram: idade materna igual ou superior a 35 anos em $748(78,1 \%)$ gestantes; ansiedade do casal em 53 (5,5\%); anomalia cromossômica em filho anterior em 39 (4,1\%); malformação fetal ao exame ultra-sonográfico em 29 (3\%); anomalias cromossômicas em progenitores em quatro $(0,4 \%)$; diagnóstico de paternidade em duas $(0,2 \%)$; doença metabólica fetal em filho anterior em uma $(0,1 \%)$; e outras (consangüinidade, história de natimorto, doenças gênicas) em 82 (8,6\%). Quinhentos e vinte e cinco $(54,8 \%)$ gestantes não tinham relato de filhos vivos quando realizaram o exame. Nos antecedentes obstétricos, abortamento espontâneo foi observado em $21,7 \%$ (208 casos) e óbito fetal em $0,6 \%$ (três casos). A maioria, 932 casos $(97,2 \%)$, não relatava nenhum abortamento espontâneo prévio. A BVC foi realizada por um único obstetra, entre a nona e a $24^{\mathrm{a}}$ semana de gestação, por via transabdominal e extra-amniótica.

$\mathrm{Na}$ anamnese realizada na consulta inicial eram obtidos a identificação da paciente, antecedentes pessoais e familiares, antecedentes obstétricos e indicação para a realização da BVC. Nesta ocasião, eram apresentados à paciente ou ao casal detalhes da técnica, seus riscos, possibilidade de repetição do exame e informações sobre o diagnóstico citogenético. Finda a entrevista, a paciente era encaminhada para aconselhamento genético.

Em seguida, a paciente era submetida a exame ultra-sonográfico, utilizando-se de aparelho Aloka 2000 com transdutor de 3,5 MHz (Aloka Co., Ltd., Tokyo, Japan), para confirmação da idade gestacional por meio da medida do CCN fetal, número de fetos, corionicidade quando aplicada, freqüência cardíaca fetal, morfologia fetal, incluindo-se a medida da translucência nucal, localização e espessura da placenta, volume subjetivo de líquido amniótico e malformações uterinas. Normalmente, não é necessário que a bexiga da paciente esteja cheia, salvo em situações de acentuada anteflexão uterina para corrigir a sua angulação ou afastar alças intestinais do trajeto da agulha.

Após lavagem rigorosa das mãos com clorexidina a 2\% (Rioquímica Indústria Farmacêutica, São Paulo) e uso de luvas cirúrgicas, procedia-se à anti-sepsia da parede abdominal com o mesmo degermante e colocação de campo cirúrgico fenestrado estéril. Não realizamos anestesia local, o que era antecipadamente explicado a paciente, por acharmos desnecessário. $\mathrm{O}$ transdutor manuseado durante todo o procedimento por profissional experimentado e qualificado era revestido com protetor esterilizado e, em vez de utilizarmos gel condutor, empregávamos solução degermante de clorexidina a $2 \%$. O manuseio do conjunto seringa-agulha era realizado pelo operador.

Uma vez localizada a placenta, uma agulha de punção espinhal 18G 31⁄2 (Becton, Dickinson Ind. Cirúrgica Ltda., MG) era introduzida, sob orientação da ultra-sonografia, livremente, sem guia de biópsia, 1 a $2 \mathrm{~cm}$ acima da porção superior do transdutor. Quando no interior da placenta, retirava-se o mandril da agulha e adaptava-se uma seringa plástica de $20 \mathrm{~mL}$ (Becton, Dickinson Ind. Cirúrgica Ltda., PR), contendo $5 \mathrm{~mL}$ de meio de cultura (Chang medium with L-glutamine, Irvine Scientific, California, USA). Executando-se movimentos suaves de vai e vem no conjunto seringa-agulha, realizava-se aspiração por pressão negativa contínua na seringa. Antes e após o procedimento, a freqüência cardíaca fetal era observada utilizando-se o M-mode. Posteriormente, procediase à retirada da agulha e a paciente era solicitada a permanecer em repouso na mesa de exame, por período de 30 minutos. Após este intervalo, a gestante era avaliada obstetricamente, questionada sobre a ocorrência de dor, tipo e intensidade. Investigava-se a presença de sangramento vaginal e perda de líquido amniótico. Procedia-se a novo exame ecográfico, visando à vitalidade fetal, hematoma subcoriônico e medida subjetiva de líquido amniótico na câmara amnica. A paciente era instruída a retornar no caso de intercorrências como dor, febre, sangramento vaginal e perda de líquido amniótico. Consulta de seguimento era agendada após 30 dias com a mesma equipe, ou com o médico assistente da gestante.

Obtida suficiente quantidade do material, 20 a $40 \mathrm{mg}$, realizava-se a sua análise em placa de Petri esterilizada no sentido de investigar a qualidade e a quantidade do vilo corial, além de eliminar excesso de sangue materno e decídua porventura existentes, sendo enviado para análise citogenética na própria seringa utilizada para punção. Para estudos genéticos realizados em outros centros fora de Salvador, o envio era feito via Sedex 10 (Empresa Brasileira de Correios e Telégrafos), em temperatura ambiente.

Para a análise citogenética, utilizou-se a técnica de bandeamento $G^{21}$, e estudo molecular por PCR (reação de cadeia de polimerase) para os cromossomos $13,16,18,21,22$, $\mathrm{X}$ e $\mathrm{Y}^{22}$, quando havia necessidade de diagnóstico rápido. 
Complicações imediatas foram definidas como aquelas ocorridas até o segundo dia após o procedimento, incluindo sintomas referidos pela paciente como cólicas uterinas (dor tipo cólica no hipogástrio), desconforto abdominal (sensação de peso ou pressão no hipogástrio), dor no local da punção, hematoma subcoriônico, punção acidental da cavidade amniótica, amniorrexe, bradicardia fetal e sangramento vaginal. Complicações tardias eram aquelas ocorridas do terceiro ao $45^{\circ}$ dia após a BVC e incluíam: dor abdominal de qualquer característica e/ou duração, sangramento vaginal, amniorrexe, infecção, abortamento espontâneo, parto prematuro e complicações obstétricas como descolamento prematuro de placenta normalmente inserida e placenta prévia.

À época prevista para o parto, realizou-se contato telefônico com a paciente ou seu médico assistente, ou consulta aos prontuários hospitalares, visando à obtenção de dados da evolução da gravidez, parto e puerpério. Eram anotados a idade gestacional à época do parto, tipo de parturição, gênero, peso e anormalidades do recém-nascido.

Para análise dos dados, utilizou-se o programa estatístico SPSS (Statistical Package for Social Science), versão 9.0 (SPSS Inc., Chicago, Illinois). As variáveis contínuas foram descritas por meio da freqüência absoluta, média \pm desvio padrão, mediana e moda. As variáveis categóricas foram descritas pela freqüência absoluta e percentual. Para comparações de variáveis categóricas foi realizado o teste do $\chi^{2}$ ou o exato de Fisher. Na comparação das variáveis contínuas foi usado o teste $t$ de Student ou Mann-Whitney.

\section{Resultados}

A taxa de sucesso de obtenção da amostra para a análise citogenética na primeira punção foi $94,7 \%$ (907 casos). Duas e três punções foram realizadas em $5 \%$ (48 casos) e 0,3\% (três casos), respectivamente. O resultado do cariótipo foi obtido em $850(88,5 \%)$ pacientes. Em 108 (11,2\%), não foi possível obteremse os resultados dos cariótipos, devido às seguintes causas: $39(4 \%)$ não possuíam informações em seus prontuários; 48 (5\%) possuíam outras indicações de exame que não o diagnóstico de anomalias genéticas; sete $(0,7 \%)$ apresentaram contaminação bacteriana do material; $12(1,2 \%)$ tiveram acidentes de transporte do material e em duas $(0,2 \%)$, o material foi insuficiente para a realização da análise citogenética.

Em 776 (81\%) pacientes, não foi observado qualquer tipo de complicação materna ou fetal. A complicação imediata mais freqüente foi cólica uterina em 134 (14\%) gestantes, com remissão espontânea em 124 (92,5\%) casos após média de uma hora de observação. Houve necessidade do tratamento analgésico em dez $(7,4 \%)$ casos com butilbrometo de escopolamina $(10 \mathrm{mg})$ via oral, em dose única. As outras complicações imediatas estão descritas na Tabela 1. Complicações tardias foram relatadas em $32(3,3 \%)$ gestantes, sendo a mais freqüente sangramento vaginal em $16(1,6 \%)$ conforme Tabela 1.

Quinze pacientes $(1,6 \%)$ apresentaram abortamento espontâneo após o procedimento, e ocorreram três óbitos fetais de causas identificadas: dois casos $(0,2 \%)$ como complicações de síndromes hipertensivas na gravidez e um caso $(0,1 \%)$ por infecção causada por Parvovírus B19 (Figura 1).

Quando comparados os dois grupos de pacientes, com complicações imediatas e sem complicações imediatas, verificamos que a taxa de abortamento espontâneo foi maior nas pacientes cujos fetos apresen-

Tabela 1 - Frequiência de complicações imediatas e tardias entre as 958 pacientes submetidas à biópsia do vilo corial por via transabdominal.

\begin{tabular}{|c|c|c|}
\hline Complicações imediatas & n & $\%$ \\
\hline Cólicas uterinas & 134 & 14,0 \\
\hline Hematoma subcoriônico & 17 & 1,8 \\
\hline Punção amniótica acidental & 12 & 1,3 \\
\hline Dor da punção ou desconforto abdominal & 14 & 1,5 \\
\hline Amniorrexe & 2 & 0,2 \\
\hline Bradicardia fetal & 2 & 0,2 \\
\hline Sangramento vaginal & 1 & 0,1 \\
\hline Total & 182 & 19,1 \\
\hline \multicolumn{3}{|l|}{ Complicações tardias } \\
\hline Sangramento vaginal & 16 & 1,6 \\
\hline Dor abdominal & 13 & 1,4 \\
\hline Amniorrexe & 3 & 0,3 \\
\hline Total & 32 & 3,3 \\
\hline
\end{tabular}

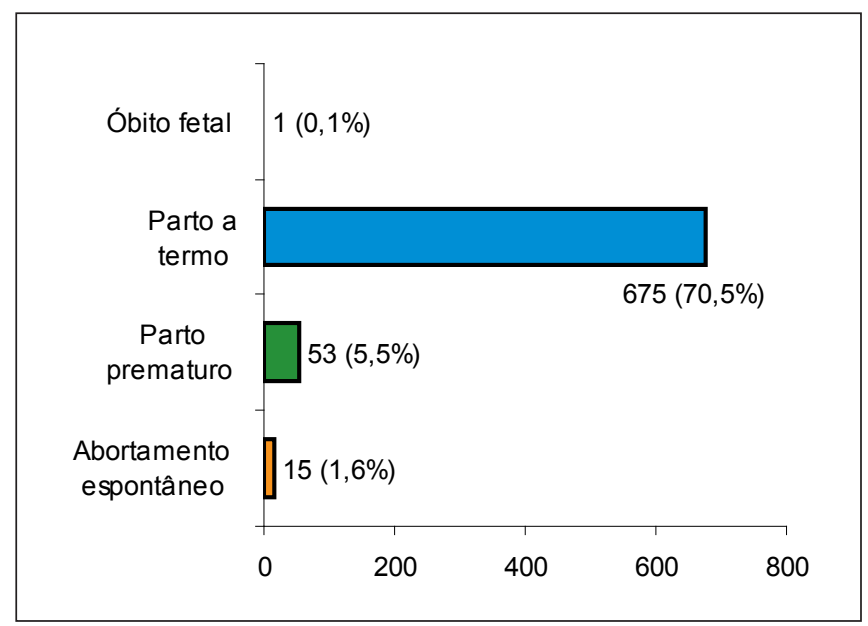

Figura 1 - Distribuição dos resultados das gestações após biópsia do vilo corial por via transabdominal em 958 pacientes. 
taram bradicardia durante a BVC $(\mathrm{p}=0,03)$. As outras complicações não estavam associadas com aumento da taxa de abortamento espontâneo (Tabela 2).

Comparando-se a idade gestacional média em que o procedimento foi realizado e interrupção da gravidez, não foi encontrada diferença estatisticamente significante entre as pacientes que evoluíram com abortamento espontâneo e as que não tiveram abortamento $(12,53 \pm 1,06$ e $12,51 \pm 1,08$, respectivamente; $\mathrm{p}=0,93$ ).

Excluindo-se os casos sem informação sobre o resultado final da gestação $(\mathrm{n}=188,19,6 \%)$, a taxa de gestação a termo foi $87,7 \%$ (675 pacientes), parto prematuro 6,9\% (53) e interrupção total da gravidez (intencional e espontânea), 5,5\% (42), conforme Figura 1.

Nenhum caso desenvolveu infecção no sítio cirúrgico (pele, subcutâneo, peritônio, útero, placenta ou âmnio), descolamento prematuro de placenta e placenta prévia. A avaliação dos recém-nascidos não mostrou ocorrência de malformações fetais.

\section{Discussão}

A BVC permite coletar células trofoblásticas oriundas do trofoectoderma contidas no vilo corial, que possui a mesma origem genética fetal. Por se dividirem muito rapidamente, estas células permitem o diagnóstico citogenético em poucos dias, tornando a BVC um procedimento mais precoce que a $\mathrm{AMC}$ e que pode ser realizado a partir da nona semana de gravidez ${ }^{1}$.

A BVC é um procedimento que vem sendo realizado há mais de 20 anos e é indicada principalmente por idade materna avançada (igual ou superior a 35 anos), devido ao risco de alterações cromossômicas aumentarem com a idade cronológica da paciente, embora a maioria das aneuploidias ocorra em gestantes abaixo dos 35 anos de idade ${ }^{3,6}$. A decisão da realização da presente análise pareceu-nos pertinente pelas evidências do aumento de indicação de BVC, como consequiência da inclusão de todas as gestantes no processo de rastreamento de trissomias fetais no prénatal, principalmente da trissomia do cromossomo 21 , independente da existência de fatores de riscos ${ }^{23}$.
Com introdução e universalização dos métodos de rastreamento pré-natal de aneuploidias a todas as gestantes, há uma tendência a diminuir o número relativo de pessoas com idade acima de 35 anos que estão sendo encaminhadas para a realização de AMC e BVC. Contudo, a idade materna avançada continua sendo a principal indicação de exames invasivos no pré-natal para o diagnóstico de anormalidades genéticas, conforme observado na atual série $(78,1 \%$ das gestantes tinham idade igual ou superior a 35 anos). Dados semelhantes foram encontrados em uma série de 10.741 gestantes na França (media de idade de $37,3 \pm 4,7$ anos e mediana de 36 anos) ${ }^{24}$ e em estudo multicêntrico envolvendo 1.914 gestantes (média de idade: $37,0 \pm 2,3$ anos) submetidas a BVC-TA ${ }^{11}$. Todavia, idades mais avançadas (média de idade: $39,3 \pm 2,6$ anos) já foram descritas em estudo brasileiro com 1.290 casos realizado entre os anos de 1985 e $1991^{20}$. Comparandose os estudos citados, observa-se tendência à diminuição da idade materna entre as pacientes submetidas à BVC em períodos mais recentes.

Neste estudo destaca-se o achado do alto percentual $(54,8 \%)$ de pacientes sem antecedentes de filhos vivos na época do procedimento. Este fato deve ser considerado no momento da indicação do exame, visto que a interrupção da gravidez faz parte das complicações $\mathrm{da} \mathrm{BVC}^{17,20}$, embora existam outros fatores que, independente da relação direta com a BVC, influenciam nas taxas de abortamento. Assim, muitos fetos com translucência nucal alterada, importante indicação de BVC, são portadores de defeitos congênitos graves, não cromossômicos ${ }^{25}$.

$\mathrm{Na}$ análise dos antecedentes obstétricos da casuística apresentada foi encontrado um elevado percentual de abortamentos espontâneos, 21,7\% (208 casos), muito provavelmente associado à idade cronológica materna avançada, é um fator de risco para abortamento espontâneo, conforme tem sido relatado na literatura ${ }^{26}$.

Em 776 (81\%) gestantes, o que representa mais de dois terços dos casos, não encontramos qualquer tipo de complicação. Entre as que apresentaram complicações, a complicação imediata mais freqüente foi

Tabela 2 - Avaliação do percentual de abortamento espontâneo em pacientes com complicações imediatas comparado com pacientes sem complicações imediatas*.

\begin{tabular}{lcccc}
\hline Complicações & $\mathrm{n}$ & Aborto espontâneo & Percentual de aborto & P \\
\hline Cólica uterina & 134 & 2 & 1,5 & 0,66 \\
Hematoma subcoriônico & 17 & 1 & 5,9 & 0,19 \\
Punção ammniótica acidental & 12 & 1 & 8,3 & 0,14 \\
Desconforto ou dor abdominal & 14 & 1 & 7,1 & - \\
Amniorrexe & 2 & - & 50 & - \\
Bradicardia fetal & 2 & 1 & - & 0,03 \\
Sangramento vaginal & 1 & - & - & - \\
\hline
\end{tabular}

*O percentual de abortamento de pacientes que não tiveram complicação imediata foi 1,2\% (9 de 776). 
dor espasmódica, do tipo cólica uterina em 14\%, facilmente controlada com repouso por tempo curto ou uso de analgésicos. Dor durante a realização da punção abdominal ou desconforto abdominal foi referido por 14 $(1,5 \%)$ gestantes. A percepção materna de dor durante o procedimento tem sido referida como importante em diversas publicações, e consideramos um fator de resistência por parte da mulher à aceitação do método. Aplicando a escala visual analógica em 300 gestantes antes e depois de procedimentos invasivos (AMC, BVC-TA e BVC-TC), nas quais não foram utilizadas medicações anestésicas, um estudo demonstrou que a dor referida antes do exame era maior que a percebida após a sua realização ${ }^{27}$. Os autores concluem haver existência de correlação positiva entre o nível de ansiedade e a dor induzida pelo ato operatório ${ }^{27}$. Estas observações justificam, na nossa experiência, a não utilização de anestésico local para a realização da punção.

Houve apenas um caso $(0,1 \%)$ de sangramento vaginal de pequena intensidade, interrompido espontaneamente em um dia, e dois casos $(0,2 \%)$ de perda de pequena quantidade de líquido amniótico, que tiveram resolução espontânea após um período de repouso no leito de 24 horas. Sangramento vaginal tipo manchas foi relatado em $4,5 \%$ em uma série de casos e não estava associado com abortamento espontâneo pós-procedimento ${ }^{17}$.

Em estudo colaborativo randomizado canadense comparando BVC e AMC, foram encontradas taxas de 2,7, 26,6 e 1,4\% de cólica abdominal, sangramento vaginal e perda de líquido amniótico, respectivamen$\mathrm{te}^{28}$. Em uma série de casos envolvendo 1.844 pacientes submetidas à BVC-TA, o sangramento vaginal ocorreu em $33(1,8 \%)$ dos $\operatorname{casos}^{1}$.

Nos $12(1,3 \%)$ casos de punção amniótica acidental, ocorreu perda de líquido amniótico em dois casos $(0,2 \%)$, ambos tratados apenas com repouso no leito e evoluindo ao termo sem ocorrência de outras complicações. O estudo colaborativo canadense antes citado apresentou freqüência de $1,4 \%$ de amniorrexe após $\mathrm{BVC}^{28}$.

Hematoma subcoriônico ocorreu em 17 (1,8\%) casos, com tamanho inferior a $2 \mathrm{~cm}$ em seu maior comprimento (dados não demonstrados) que tiveram resolução espontânea. Todos foram acompanhados com exames ultrasonográficos semanais e desapareceram completamente em até duas semanas após a realização da BVC.

A conduta preconizada nestes casos era que a paciente obedecesse a repouso de atividades físicas e sexuais, retornando semanalmente para avaliação ultra-sonográfica. Em todos os casos, houve regressão espontânea e não estiveram associados a complicações como abortamento ou sangramento vaginal. Em estudo realizado no Paquistão entre 143 gestantes submetidas à BVC-TA, a maioria para diagnóstico de -talassemia entre 12 e 14 semanas, foi relatado hematoma subcoriônico em 4,9\% (sete de 143 pacientes) ${ }^{29}$. Três cursaram com pequeno sangramento vaginal após o procedimento, duas tiveram regressão espontânea após três a seis horas de repouso hospitalar, enquanto uma paciente evoluiu com abortamento espontâneo após seis horas. $\mathrm{O}$ autor não descreve o volume do hematoma, sendo provável que os mais volumosos possam evoluir para descolamento da placenta e abortamento subseqüente.

As taxas de abortamento espontâneo $(1,6 \%)$ e óbitos fetais $(0,3 \%)$ em nossa casuística foram semelhantes àquelas descritas em estudo não randomizado, no qual foi encontrada taxa de abortamento espontâneo de $1,65 \%$ e perdas fetais de $0,5 \%$ em 665 gestantes submetidas à BVC-TA ${ }^{18}$. Taxa de abortamento espontâneo de 8,4\% (346 casos) foi citada entre pacientes que não realizaram o procedimento ${ }^{18}$. Em outra publicação, os autores relataram taxa de 1,4\% (142 casos) de abortamento espontâneo e 0,3\% (31 casos) de perda gestacional em 9.352 BVC-TA realizadas depois da $11^{\text {a }}$ semana de gestação ${ }^{24}$. Posteriormente, em estudo de série de casos $(\mathrm{n}=9.886)$ de BVC-TA e BVC-TC, sem separar os resultados por técnica de exame, foi relatada um taxa total de perdas fetais de

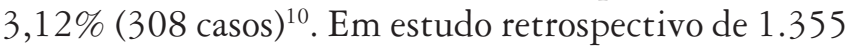
casos de BVC-TA, principalmente por idade materna avançada, realizados por cinco diferentes operadores de um mesmo serviço na China, entre 11 e 13 semanas, foram encontrados $0,73 \%$ (nove casos) de perdas fetais associadas a causas obstétricas independentes e $1,54 \%$ (dez casos) potencialmente decorrentes do procedimento $^{30}$. Como a taxa de perdas fetais em grupo controle de pacientes não expostas foi $0,8 \%$, os autores concluíram que a taxa de perdas fetais relacionadas ao exame foi de $0,74 \%{ }^{30}$.

$\mathrm{Na}$ análise das complicações imediatas, apenas a bradicardia fetal esteve associada com maior percentual de abortamento. Bradicardia fetal tem sido relacionada com aborto espontâneo, sendo recomendado retardar a realização da BVC para observar a evolução da gestação pelo risco de óbito fetal nesses $\operatorname{casos}^{31}$. Recentemente foram descritas alterações da freqüência cardíaca fetal em estudo com 300 gestantes submetidas à BVC-TA entre a oitava e $13^{\text {a }}$ semanas, sugerindo alterações hemodinâmicas fetais, significativamente associadas à idade gestacional e idade materna e sem associações com a duração do procedimento, número de inserções e sexo fetal ${ }^{32}$. São desconhecidas até o momento quais as repercussões da variação da freqüência cardíaca fetal a longo prazo. 
Em 926 (96,7\%) gestantes não ocorreram complicações tardias. Amniorrexe foi encontrada em três casos $(0,3 \%)$, parecendo estar associada com o evento, sendo que dois casos foram os mesmos relatados entre as complicações imediatas. Amniorrexe foi descrita como complicação materna em 0,86\% (17 casos) de uma série de 1984 pacientes submetidas à BVC comparado com taxa de $1,2 \%$ (583 casos) em pacientes não expostas ${ }^{15}$. Todas as pacientes da nossa série que apresentaram amniorrexe evoluíram até o termo da gestação e não apresentaram outras intercorrências obstétricas ou neonatais.

A taxa de parto prematuro foi de 5,5\% (52 casos), semelhante à encontrada $(5,4 \%, \mathrm{n}=107$ casos) em estudo de coorte realizado na Suécia, envolvendo 1.984 pacientes submetidas à BVC-TA entre 10 e 12 semanas $^{33}$.

A despeito de relato anterior de malformações fetais associadas à $\mathrm{BVC}^{34}$, não encontramos nenhum caso na nossa série. Em estudo de coorte incluindo paciente com idade variando entre 35 e 49 anos e com gravidez de feto único, foram comparados os resultados de AMC $(\mathrm{n}=21.748$ casos), BVC (1.984 casos) e grupo não exposto a procedimentos (47.854 casos), tendo sido encontradas taxas de reduções de membros fetais similares (AMC, 0,4/1000; BVC, 0,5/1000; e grupo não exposto, $0,5 / 1000)^{33}$. Há ainda observação da ausência da extremidade do terceiro quirodáctilo como possível efeito teratogênico da $\mathrm{BVC}^{35}$; todavia, as conclusões dos autores são questionadas pela pequena casuística e falta de documentação suficiente ${ }^{13}$.
Acreditamos que a baixa taxa de complicações encontrada em nossa série deva estar associada aos critérios técnicos rigorosos adotados para indicação e a realização do procedimento, à inserção única na maioria dos casos e ao fato de não ser realizada punção intra-amniótica.

Em conclusão, a BVC-TA em nossa instituição revelou-se um procedimento simples e bastante seguro, resultado da larga experiência do operador. A BVC-TA realizada no primeiro trimestre de gravidez deve ser considerada como método de escolha para o diagnóstico pré-natal de aneuploidias, devido às baixas taxas de complicações apresentadas no presente trabalho. $\mathrm{O}$ fato de ser realizado precocemente na gestação permite rápido diagnóstico em pacientes identificadas de alto risco por meio dos testes de rastreamento, contribuindo para reduzir a ansiedade da gestante. Apenas profissional bem treinado e experiente deve realizar o procedimento. Lembramos ser fundamental esclarecer à gestante, ou ao casal preferencialmente, que o procedimento envolve riscos, embora de baixa freqüência.

\section{Agradecimentos}

Doutora Ingrid Brandão, Doutora Vecirley Macedo, Doutora Luciana Vieira Lopes, Doutor José Tavares Neto, Professor Doutor Edgar Carvalho, Senhora Darlene Pimentel e Senhora Maria Luiza Eirado Lima.

\section{Referências}

1. Brambati B, Tului L, Camurri L, Guercilena S. Early second trimester (13 to 20 weeks) transabdominal chorionic villus sampling (TACVS): a safe and alternative method for both high and low risk populations. Prenat Diagn. 2002; 22(10):907-13.

2. Chiu RW, Lo YM. Noninvasive prenatal diagnosis by analysis of fetal DNA in maternal plasma. Methods Mol Biol. 2006; 336:101-9.

3. Snijders R, Sundberg K, Holzgreve W, Henry G, Nicolaides KH. Maternal age- and gestation-specific risk for trisomy 21. Ultrasound Obstet Gynecol. 1999; 13(3): 167-70.

4. Brambati B, Tului L, Cislaghi C, Alberti E. First 10,000 chorionic villus sampling performed on singleton pregnancies by a single operator. Prenat Diagn. 1998; 18(3):255-66.

5. Papp C, Beke A, Mezei G, Tóth-Pál E, Papp Z. Chorionic villus sampling: a 15-year experience. Fetal Diagn Ther. 2002; 17(4):218-27.

6. Brambati B, Tului L, Simoni G, Travi M. Genetic diagnosis before the eighth gestational week. Obstet Gynecol. 1991; 77(2):318-21.

7. MRC Working Party on the Evaluation of Chorion Villus Sampling. Medical Research Council European trial of chorion villus sampling. Lancet. 1991; 337(8756): 1491-9.
8. Alfirevic Z. Early amniocentese versus transabdominal chorion villus sampling for prenatal diagnosis. Cochrane Database Syst Rev. 2002; (4):CD000077.

9. Smidt-Jensen S, Philip J, Lundsteen C, Permin M, Zachary JM, Fowler SE. Randomised comparison of amniocentesis and transabdominal and transcervical chorionic villus sampling. Lancet. 1992; 340(8830): 1237-44.

10. Caughey $A B$, Hopkins $L M$, Norton ME. Chorionic villus sampling compared with amniocentesis and the difference in the rate of pregnancy loss. Obstet Gynecol. 2006; 108(3Pt 1):612-6.

11. Philip J, Silver RK, Wilson RD, Thom EA, Zachary JM, Mohide $P$, et al. Late first- trimester invasive prenatal diagnosis: results of an international randomized trial. Obstet Gynecol. 2004; 103(6): 11 164-73.

12. Boyd PA, Keeling JW, Selinger M, MacKenzie IZ. Limb reduction and chorion villus sampling. Prenat Diagn. 1990; 10(7):437-41.

13. Brambati B, Tului L. Chorionic villus sampling and amniocentesis. Curr Opin Obstet Gynecol. 2005; 17(2):197-201.

14. Froster UG, Jackson L. Limb defects and chorionic villus sampling: results from an international registry, 1992-1994. Lancet. 1996; 347(9000):489-94. 
15. Cederholm M, Haglund B, Axelsson O. Maternal complications following amniocentesis and chorionic villus sampling for prenatal karyotyping. BJOG. 2003; 110(4):392-9.

16. Monni G, Zoppi MA, Axiana C, Ibba RM. Changes in the approach for invasive prenatal diagnosis in 35,127 cases at a single center from 1977 to 2004. Fetal Diagn Ther. 2006; 21 (4):348-54.

17. Williams J 3rd, Wang BB, Rubin $\mathrm{CH}$, Aiken-Hunting D. Chorionic villus sampling: experience with 3016 cases performed by a single operator. Obstet Gynecol. 1992; 80(6):1023-9.

18. Scott F, Peters H, Boogert T, Robertson R, Anderson J, Mclennan A, et al. The loss rates for invasive prenatal testing in a specialised obstetric ultrasound practice. Aust N Z J Obstet Gynecol. 2002; 42(1):55-8.

19. Fonseca ALAF, Amin Júnior J, Chaves Netto H, Montenegro CAB. Biopsia de vilo corial por via transabdominal. J Bras Ginecol. 1990; 100(5/6):111-9.

20. Gollop TR, Naccache NF, Campos IMA, Pieri PC. Amostra de vilo corial: 1290 casos. Rev Bras Ginecol Obstet. 1993; 15(2):84-7.

21. Sanchez $\mathrm{O}$, Escobar JI, Yunis JJ. A simple G-banding technique. Lancet. 1973; 3022(7823):269.

22. Van den Berg C, Van Opstal D, Brandenburg H, Wildschut HI, den Hollander NS, Pijpers L, et al. Accuracy of abnormal karotypes after the analysis of both short- and long-term culture of chorionic villi. Prenat Diagn. 2000; 20(12):956-69.

23. Nicolaides KH, Spencer K, Avgidou K, Faiola S, Falcon O. Multicenter study of first-trimester screening for trisomy 21 in 75,821 pregnancies: results and estimation of the potential impact of individual risk-orientated two-stage first-trimester screening. Ultrasound Obstet Gynecol. 2005; 25(3):22 1-6.

24. Brun JL, Mangione R, Gangbo F, Guyon F, Taine L, Roux D, et al. Feasibility, accuracy and safety of chorionic villus sampling: a report of 10741 cases. Prenat Diagn. 2003; 23(4):295-301.

25. Souka AP, Snijders R, Novakov A, Soares W, Nicolaides KH. Defects and syndromes in chromosomally normal fetuses with increased nuchal translucency thickness at 10-14 weeks of gestation. Ultrasound Obstet Gynecol. 1998; 11 (6):391-400.
26. Wilson RD, Kendrick V, Witttmann BK, McGillivray B. Spontaneous-abortion and pregnancy outcome after normal first- trimester ultrasound examination. Obstet Gynecol. 1986; $67(3): 352-5$

27. Vandenbossche F, Horovitz J, Guyon F, Verret C, Saura R. Pain experience during chorionic villus sampling and amniocentesis: A preliminary study. Eur J Obstet Gynecol Reprod Biol. 2007 May 10; [Epub ahead of print].

28. Lippmann A, Tomkins DT, Shine J, Hamerton JL. Canadian multicenter randomized clinical trial of chorionic villus sampling and amniocentesis: final report. Prenat Diagn. 1992; 12(5):385-408.

29. Ahmed S. Transabdominal chorionic villus sampling (CVS) for prenatal diagnosis of genetic disorders. J Coll Physicians Surg Park. 2006; 16(3):204-7.

30. Lau TK, Leung YT, Fung YT, Chan LW, Sahota DS, Leung NT. Outcome of 1,355 consecutive transabdominal chorionic villus samplings in 1,351 patients. Chin Med J (Engl). 2005; $118(20): 1675-81$.

31. Yagel S, Anteby E, Ron M, Hochner-CelnikierD, Achiron R. The role of abnormal fetal heart rate in scheduling chorionic villus sampling. Br J Obstet Gynecol. 1992; 99(9):739-40.

32. Radunovic N, Kuczynski E, Kontic O, Kanjuh V, Lockwood CJ. Chorionic villus sampling significantly affects fetal cardiocascular function. J Matern Fetal Neonatal Med. 2007; 20(4):285-8.

33. Cederholm M, Haglund B, Axelsson O. Infant morbidity following amniocentesis and chorionic villus sampling for prenatal karyotyping. BJOG. $2005 ; 112(4): 394-402$

34. Evaluation of chorionic villus sampling safety: WHO/PAHO consultation on CVS. Prenat Diagn. 1999; 19(2):97-9.

35. Golden CM, Ryan LM, Holmes LB. Chorionic villus sampling: a distinctive teratogenic effect on fingers? Birth Defects Res A Clin Mol Teratol. 2003; 67(8):557-62. Comment in: Birth Defects Res A Clin Mol Teratol. 2004; 70(3):153; author reply 154. 